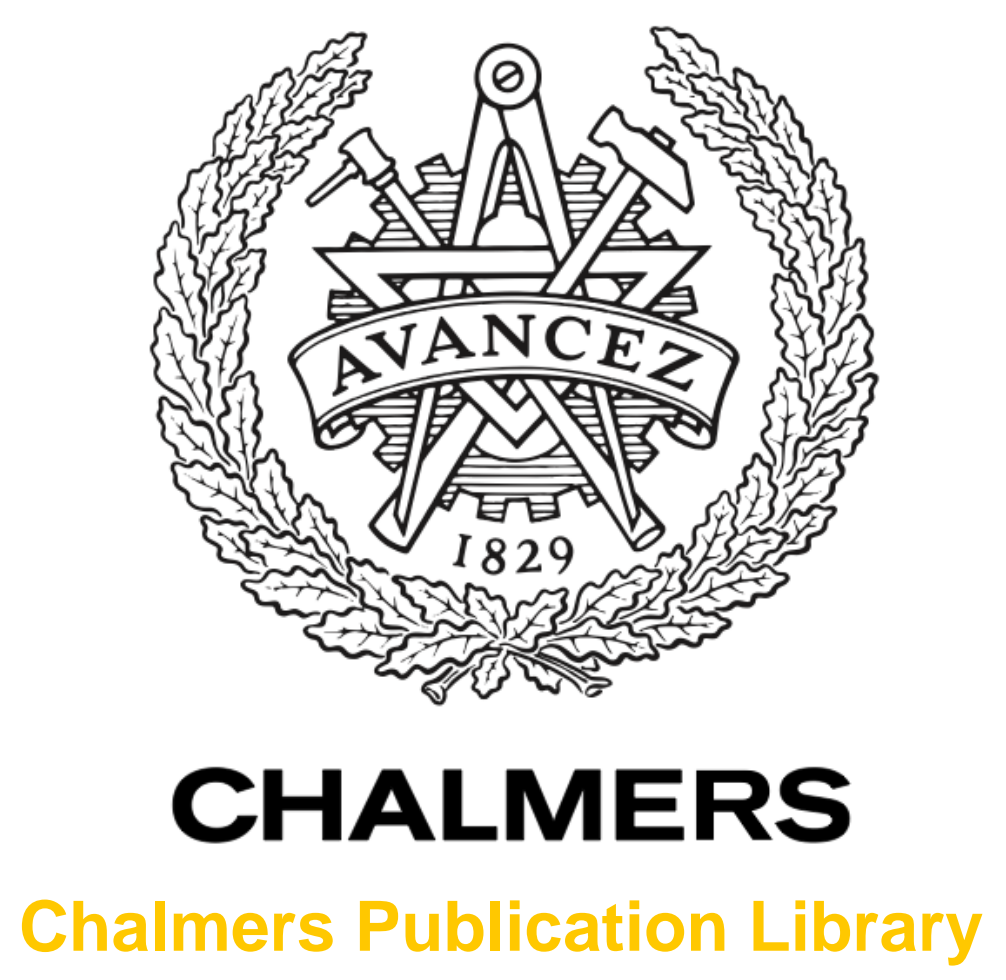

To be or not to be asymmetric? VLTI and the mass loss geometry of red giants

This document has been downloaded from Chalmers Publication Library (CPL). It is the author's version of a work that was accepted for publication in:

Proceedings of SPIE - Optical and Infrared Interferometry III, Amsterdam, Netherlands, 1-6 July 2012 (ISSN: 0277-786X)

Citation for the published paper:

Paladini, C. ; Klotz, D. ; Sacuto, S. (2012) "To be or not to be asymmetric? VLTI and the mass loss geometry of red giants". Proceedings of SPIE - Optical and Infrared Interferometry III, Amsterdam, Netherlands, 1-6 July 2012, vol. 8445

http://dx.doi.org/10.1117/12.926380

Downloaded from: http://publications.lib.chalmers.se/publication/174075

Notice: Changes introduced as a result of publishing processes such as copy-editing and formatting may not be reflected in this document. For a definitive version of this work, please refer to the published source. Please note that access to the published version might require a subscription. 


\title{
To be or not to be asymmetric? VLTI and the mass loss geometry of red giants
}

\author{
Claudia Paladini ${ }^{a}$, Daniela $\operatorname{Klotz}^{a}$, Stephane Sacuto $^{b}$, Josef $\operatorname{Hron}^{a}$, Markus Wittkowski ${ }^{c}$, Eric \\ Lagadec $^{c}$, Tijl Verhoelst ${ }^{d}$, Alain Jorissen ${ }^{e}$, Andrea Richichi ${ }^{f}$, Martin Groenewegen ${ }^{g}$, Hans \\ Olofsson $^{h}$, and Franz Kerschbaum ${ }^{a}$. \\ ${ }^{a}$ University of Vienna, Department of Astrophysics, Türkenschanzstrasse 17, 1180 Wien, \\ Austria; \\ ${ }^{b}$ University of Uppsala, Department of Physics and Astronomy, Division of Astronomy and \\ Space Physics, Box 516, 75120, Uppsala, Sweden; \\ ${ }^{c}$ ESO, Karl-Schwarzschild-Str. 2, 85748 Garching bei München, Germany; \\ ${ }^{d}$ Belgian Institute for Space Aeronomy (BIRA-IASB), Ringlaan-3-Avenue Circulaire, B-1180 \\ Brussels, Belgium; \\ ${ }^{e}$ Institut dAstronomie et dAstrophysique, Université libre de Bruxelles, CP 226, Boulevard du \\ Triomphe, 1050 Bruxelles, Belgium;

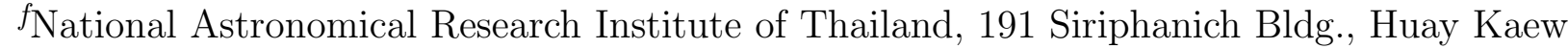 \\ Rd., Suthep, Muang, 50200 Chiang Mai, Thailand; \\ ${ }^{g}$ Koninklijke Sterrenwacht van België, Ringlaan 3, 1180 Brussel, Belgium; \\ ${ }^{h}$ Onsala Space Observatory, Dept. of Earth and Space Sciences, Chalmers University of \\ Technology, 43992 Onsala, Sweden
}

\begin{abstract}
The mass-loss process is a key ingredient for our understanding in many fields of astrophysics, including stellar evolution and the enrichment of the interstellar medium (ISM) via stellar yields. We combined the capability of the VLTI/MIDI and VLT/VISIR instruments with very recent Herschel/PACS observations to characterize the geometry of mass loss from evolved red giants on the Asymptotic Giant Branch (AGB) at various scales. This paper describes the sample of objects, the observing strategy, the tool for the interpretation, and preliminary MIDI results for two targets: U Ant and $\theta$ Aps.
\end{abstract}

Keywords: optical interferometry, infrared, imaging, stellar atmospheres, late-type stars, mass-loss, Herschel

\section{INTRODUCTION}

In the last years radio and interferometric observations were often used to investigate the history of the mass-loss. Those observations evidenced that the wind mechanism may be different for an early and a late AGB-star, a low- and a high-mass object, during and between the thermal pulse. Despite first evidences in favor of spherical symmetry, some observations show very complicated geometry and no consensous has been reached (Ref. 1).

For O-rich stars, observations of $\mathrm{SiO}$ masers emitting at few stellar radii from the stars show a general evidence of isotropic mass-loss. Water and $\mathrm{OH}$ masers are less conclusive in comparison to the $\mathrm{SiO}$ results, nevertheless no obvious departure from spherical symmetry was detected so far (Ref. 2,3). All of these works include only assumptions on the position and the size of the central sources, since at that time the high angular resolution facilities were still under development.

Going deeper inside the circumstellar envelope of the star, at a distance of around 10 stellar radii, asymmetries, clumpiness, and time-variable mass-loss have been detected (Ref. 4,5). A significant fraction of the CO line

Further author information: (Send correspondence to C.P.)

C.P.: E-mail: claudia.paladini@univie.ac.at, Telephone: +43-1-4222-53853

Optical and Infrared Interferometry III, edited by Françoise Delplancke, Jayadev K. Rajagopal, Fabien Malbet, Proc. of SPIE Vol. 8445, 84451R - (C) 2012 SPIE · CCC code: 0277-786/12/\$18 - doi: 10.1117/12.926380 
profiles obtained toward M-stars deviate significantly from those expected from a spherical envelope (Ref. 6). Different scenarios have been proposed to explain this kind of profiles involving a very slow wind in spherical expansion and a faster bipolar flow (Ref .7), 2 successive winds of different ages with different expansion velocities (Ref. 8), a long-lived reservoir of orbiting molecular gas in Keplerian rotation (Ref. 9). or a binary interaction (Ref. 10). A last interpretation could come from a possible interaction of the circumstellar environment with the ISM. Unfortunately the quality of the data used for these studies did not allow to obtain unique solutions for the structures. A synergy with IR interferometric observations is necessary to disentangle the problem.

For the C-stars there is no counterpart of $\mathrm{SiO}$, water, or $\mathrm{OH}$ maser emission, therefore the inner part of the CSE could not be properly probed by using line emission. Observations on the CO radio emission lines revealed a spatially resolved CSE in the form of spherically symmetric thin shells (Ref. 11). Up to now only a very low percentage of stars showed deviation from sphericity in this region. Nevertheless, the images of the dusty region within 10 stellar radii show highly inhomogeneous distribution due to clumpy structures (Ref. 12,13).

With its improved sensitivity (compared to the past missions), and extension to unexplored wavelength regions, the Herschel/PACS instrument is currently giving a significant contribution in understanding the massloss and dust formation processes. The Herschel Mass-loss of Evolved StarS guaranteed time key program MESS (Ref. 14) investigates the dust and gas chemistry and the properties of CSEs for a large, representative sample of post-main-sequence objects using both imaging and spectroscopy. Early MESS results (Ref. 15-17) give now for the first time a more general picture. (a) The correlation of the thermal emission of dust and gas out to large stellar distances is striking in most of the cases, putting severe limits to a possible drift between dust and gas. (b) The spatial distribution of the dusty material can be categorized in 3 to 4 forms: (1) perfectly spherical (sometimes detached) material, (2) bow shock structures correlated with the stellar motion with respect to the surrounding ISM, (3) mixtures of both and (4) influences of binarity. Clumpiness is seen on all scales. Altogether it is clear that one has to probe all spatial scales to understand the physics of these complex outflows - from the immediate stellar environment out to the Outflow/ISM interface.

In September 2010 we proposed ESO a Large Program (PI. Paladini) with the aim to complement the Herschel observations with VLTI/MIDI and VISIR observations. The program was accepted at the beginning of 2011, and we are currently in the final stage of the MIDI data analysis. The VISIR time was lost because of exceptionally bad weather conditions, but it was granted in open time for the next period of observations.

In this contribution we will show that the synergy of the three instruments represents a unique possibility to trace and constrain the origin of asymmetries in the atmosphere of AGB stars at different wavelength and spatial scales.

The VLTI/MIDI facility, thanks to the high angular resolution, is currently the most powerful tool for studying the close circumstellar environments of AGB stars where dust is forming. VISIR's angular resolution and spectral coverage are located right in between Herschel and MIDI, thus allowing to probe the intermediate region of the circumstellar environment. MIDI and VISIR observations combined with Herschel/PACS are a unique chance to have a complete picture of the different stages of the mass-loss process. Moreover they will furnish empirically based input to improve our understanding of the mass-loss process, and thus give better constraints for the next generation of $2 \mathrm{D}$ and $3 \mathrm{D}$ model atmospheres.

\section{TARGETS AND OBSERVING STRATEGY}

\subsection{Targets}

The targets of our project are a sub-sample of the AGB stars observed within the Herschel key program MESS. Beside obvious criteria such as observability from Paranal, object not included in any Guarantee Time Observations (GTO) list, and brightness within the limits of the instrument, the targets were selected to have different chemistries and variability types. To do this we used as reference the IRAS two-color diagram (Ref. 18), and we tried to sample it homogeneously as shown in Fig. 1. In this way we will be able to follow the evolution of a star along the AGB. According to the evolutionary track the objects evolve from the bottom left box to the upper right box. In Box I O-rich non variable stars with no extended circumstellar shells are expected. In Box II the objects are surrounded by young O-rich shells, while in Box IIIa the shells are more evolved. In Box IVa most of the C-rich objects with relatively cold dust are located, while in Box VIb the objects with hot dust close to

Proc. of SPIE Vol. 844584451 R-2 


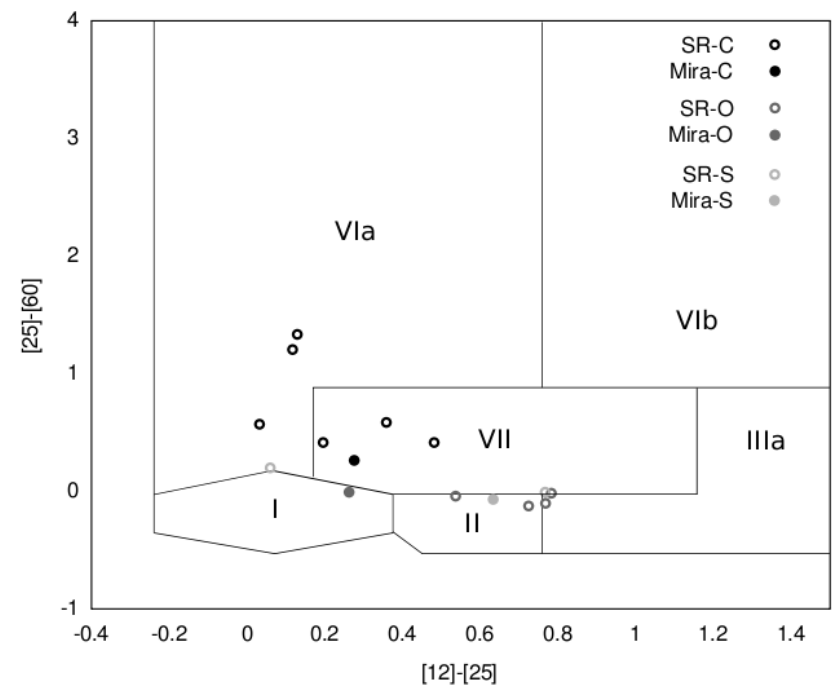

Figure 1. The targets of our sample shown in the IRAS two-color diagram (Ref. 18).

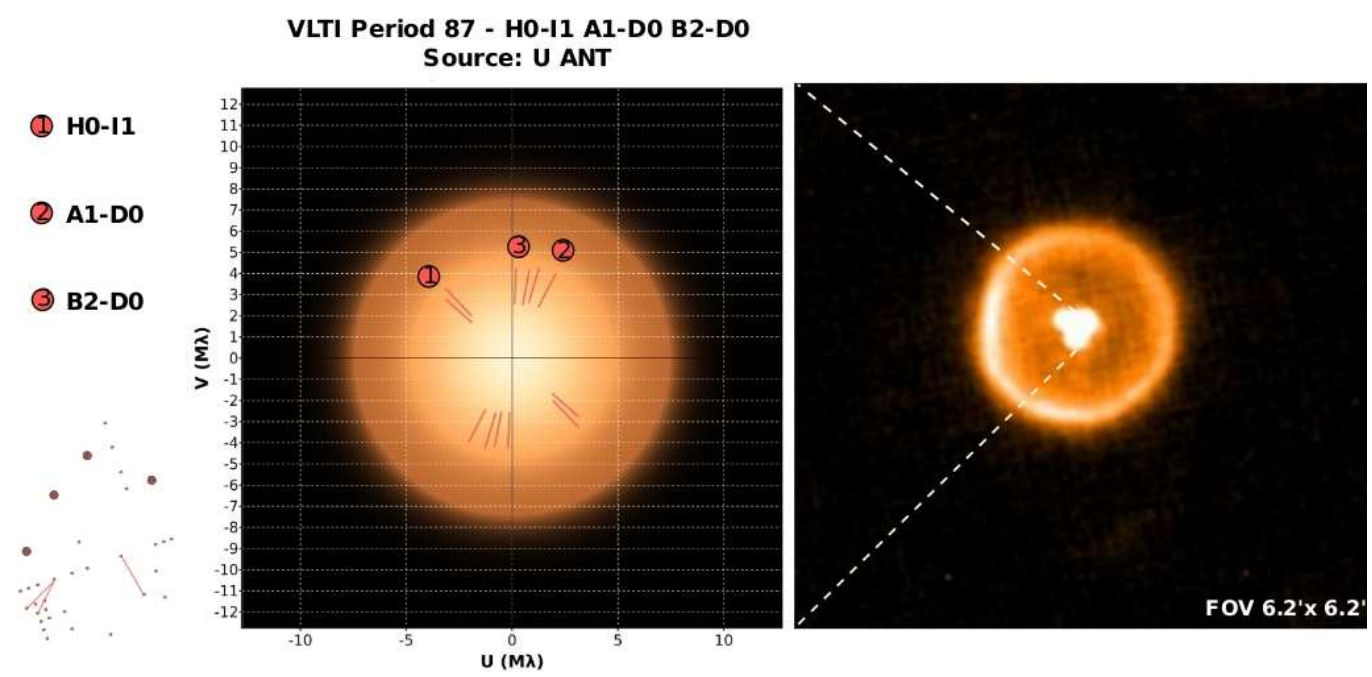

Figure 2. Left: ASPRO simulation of the object in the uv-plane. The red lines show the chosen MIDI configurations. Right: Herschel/PACS image of the star U Ant (Ref.15).

the star, and cold dust at larger distances are located. The typical variables with more evolved C-rich shells are in Box VII.

\subsection{Observing strategy}

Our targets have the advantage to be very bright in the $N$ band, therefore all the observations are carried out using MIDI with the Auxiliary Telescopes (1.8 m). The choice of the configurations was splitted in two categories:

1. Configurations for non-spherical objects evidenced by the Herschel image.

2. Configurations for spherical objects evidenced by the Herschel image (see Fig. 2).

In the first case, baselines are chosen, as good as possible (according to the availability of the proposed configurations), in the direction and perpendicular to the asymmetry seen from the Herschel image. An additional 
baseline, necessary to confirm the elongation, was chosen at a PA different than the others. A last requirement was to keep the baseline length as similar as possible to get the best reliability on the potential flatness of the structure. Also in the second case the baseline configurations were chosen with similar length and overall good uv-coverage in order to constrain any possible deviation from sphericity (see Fig. 2). For the preparation of the observations, the use of ASPRO* was crucial.

The size of the objects was estimated on the basis of the $V-K$ relation, and assuming that the dust shell (i.e. the size of the envelope in the $N$ band) is 3-4 times larger than the diameter of the object in the nearIR as expected by theoretical models.

\section{DATA REDUCTION}

The data reduction was performed using MIA+EWS ${ }^{\dagger \ddagger}$ version 1.7.1 software. Every target was bracketed between two calibrators (CAL-SCI-CAL). A detailed description of the data quality check and error determination can be found in Ref. 19 .

\section{TOOL FOR INTERPRETATION}

As the topic of our investigation is the morphology of the atmosphere of AGB stars, we developed a tool to fit the MIDI observations with geometric models. The tool is called GEM-FIND (GEometrical Model Fitting for INterferometric Data) and allows fitting the dispersed visibility measurements with central symmetric and asymmetric geometric models. The tool also derives a synthetic differential phase that can be compared with the one observed by MIDI. More details on GEM-FIND are described in Ref. 19 and Klotz et al. in this same volume. For our investigation we fitted the MIDI data with the following geometric models: circular uniform disc (UD), circular Gaussian, elliptical UD, elliptical Gaussian, binary (only in the case of more than 6 visibility points). These models are marked with letter M1, M2, M3, M4, M7 in Table 1 of Klotz et al. contribution.

\section{PRELIMINARY RESULTS}

We present here preliminary results from the MIDI campaign on two stars from our sample: U Ant and $\theta$ Aps.

\subsection{U Ant}

$\mathrm{U}$ Ant is a carbon rich Lb variable. The temperature of this target is $T_{\text {eff }}=2810 \mathrm{~K}$ (Ref. 20 . It has a $C / O=1.44$ and a mass-loss rate of $2.0 \times 10^{-6} M_{\odot} y r^{-1}$ (Ref. 21). A spherical detached shell can be seen in the Herschel-PACS image (see Fig. 4) with a distance from the star of 42" (Ref. 15). The very smooth intensity profile suggests that after a sudden increase in mass-loss (2800 years ago, Ref.22) it decreased again and did not vary much since then. The present day mass loss of the gas is $2 \times 10^{-8} M_{\odot} y r^{-1}$.

Only 2 points out of the initial sample of 6 could be used for the fitting. Three additional data points were available in the ESO archive. As the star has very small mass-loss rate, and the amplitude of pulsation is smaller than $1 \mathrm{mag}$ in $V$ band (Ref. 23), it is reasonable to consider negligible any effect of pulsation in the $N$ band interferometric observations. As a consequence, the two samples of data could be merged, and Fig. 3 shows the $\mathrm{u}-\mathrm{v}$ coverage obtained for this target.

As one can note in Fig. 4, most of the observations show an object that is almost unresolved, except for one of the archive observations taken at longer baselines. Observations were fitted with GEM-FIND and the resulting reduced $\chi^{2}$ gave very similar results for the four models tested (circular and elliptical UD and Gaussian). As one would expect it is not possible to distinguish between UD and Gaussian profile with such high visibilities (Ref. 24). The differential phase of the observations is zero for all baselines. These observations allow us to derive a size for the inner shell, and show no indication of large scale variation from spherical symmetry. The UD diameter of the object derived is $\sim 15$ mas. Indeed, the object appears around 3 times smaller than what we expected during the preparation of the observations. This kind of trend is confirmed for some other targets, and

\footnotetext{
*http://www.jmmc.fr/aspro_page.htm

${ }^{\dagger}$ http://www.strw. leidenuniv.nl/ nevec/MIDI/

${ }^{\ddagger}$ http://www.mpia-hd.mpg.de/MIDISOFT/
} 

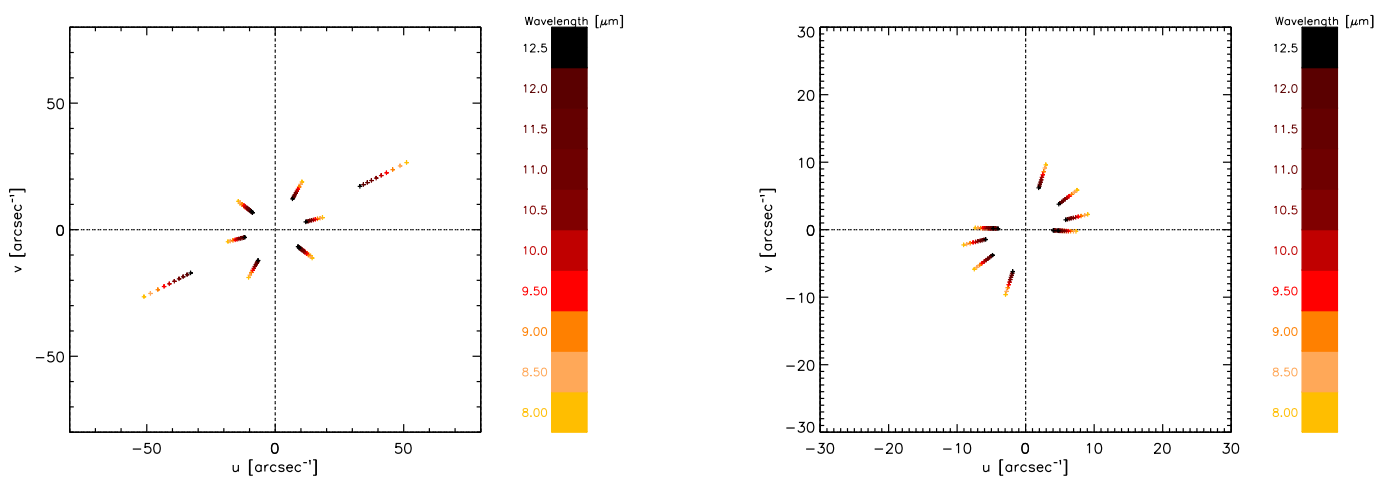

Figure 3. Color dispersed $\mathrm{u}-\mathrm{v}$ coverage for the MIDI observations of $\mathrm{U}$ Ant (left) and $\theta$ Aps (right).
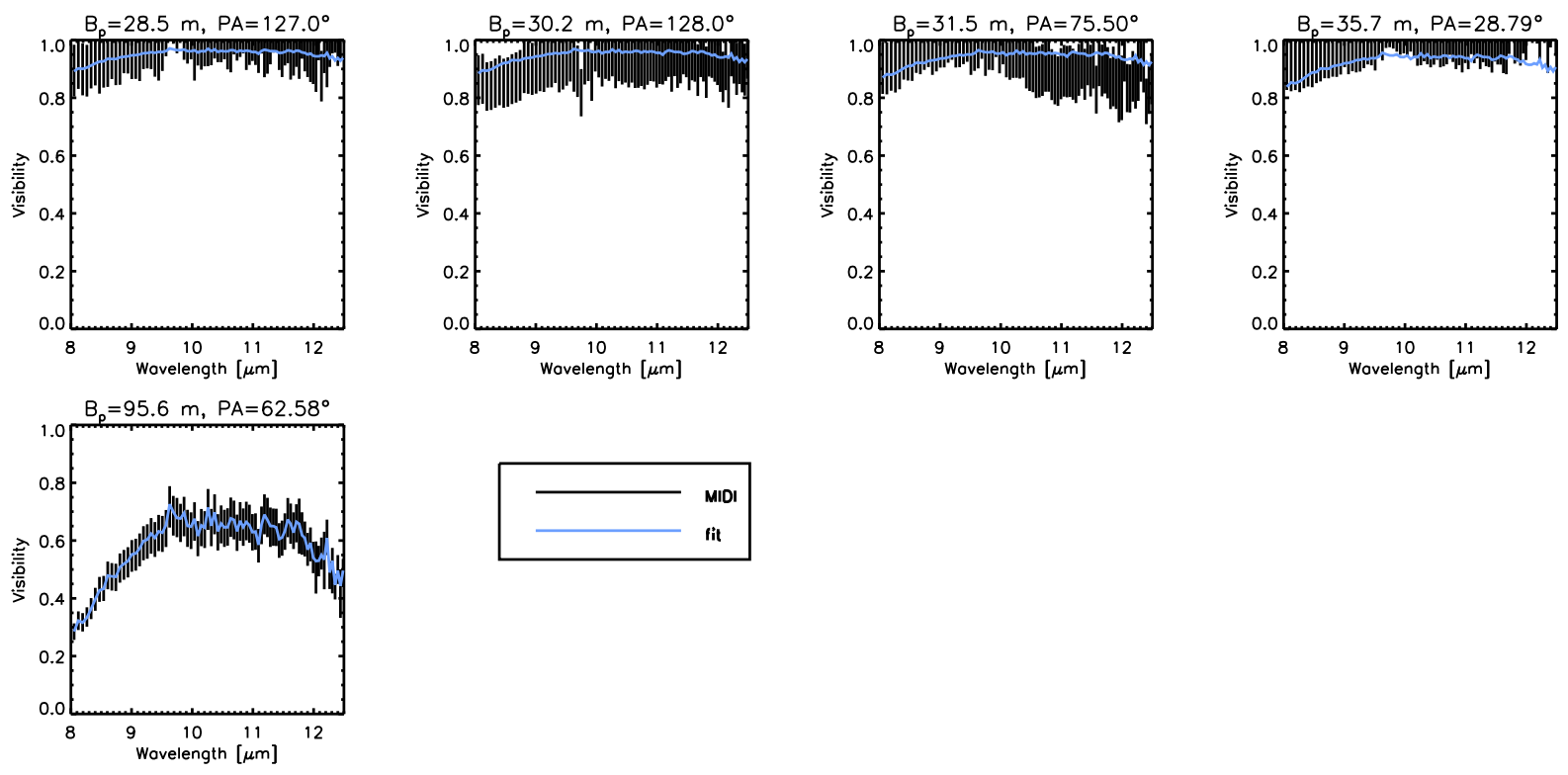

Figure 4. Visibilities dispersed in wavelength of the MIDI observations of U Ant. The full line is the best fitting Gaussian model.

we are currently in the process of discussing the implications this might have for the theory of dust formation. The visibilities dispersed in wavelength decrease around $8 \mu \mathrm{m}$, showing the typical signature of $\mathrm{C}_{2} \mathrm{H}_{2}$ molecular contribution. On the other hand, there is no trace of $\mathrm{SiC}$ dust at $11.3 \mu \mathrm{m}$. The star is probably surrounded by a very optically thin shell of amorphous carbon dust. This will be confirmed in a follow-up study with model atmospheres from Ref. 25, 26.

\section{$5.2 \theta$ Aps}

$\theta$ APS is a semiregular variable SRb with a period of 119 days. The Herschel-PACS image shows a bow shock at a distance of 68" from the center of the star (Ref. 27). Moreover, an elongation of the envelope in the perpendicular direction at around 110 degrees was detected. This O-rich star has a mass-loss rate of $4 \times 10^{-8} M_{\odot} y r^{-1}$ and a temperature of $2620 \mathrm{~K}$ (Ref. 28).

The uv-coverage of the target is shown in the right panel of Fig. 3. Five observations out of the initial 6 could be used for the geometric model-fitting. In this case, as well as for U Ant, we cannot really distinguish 

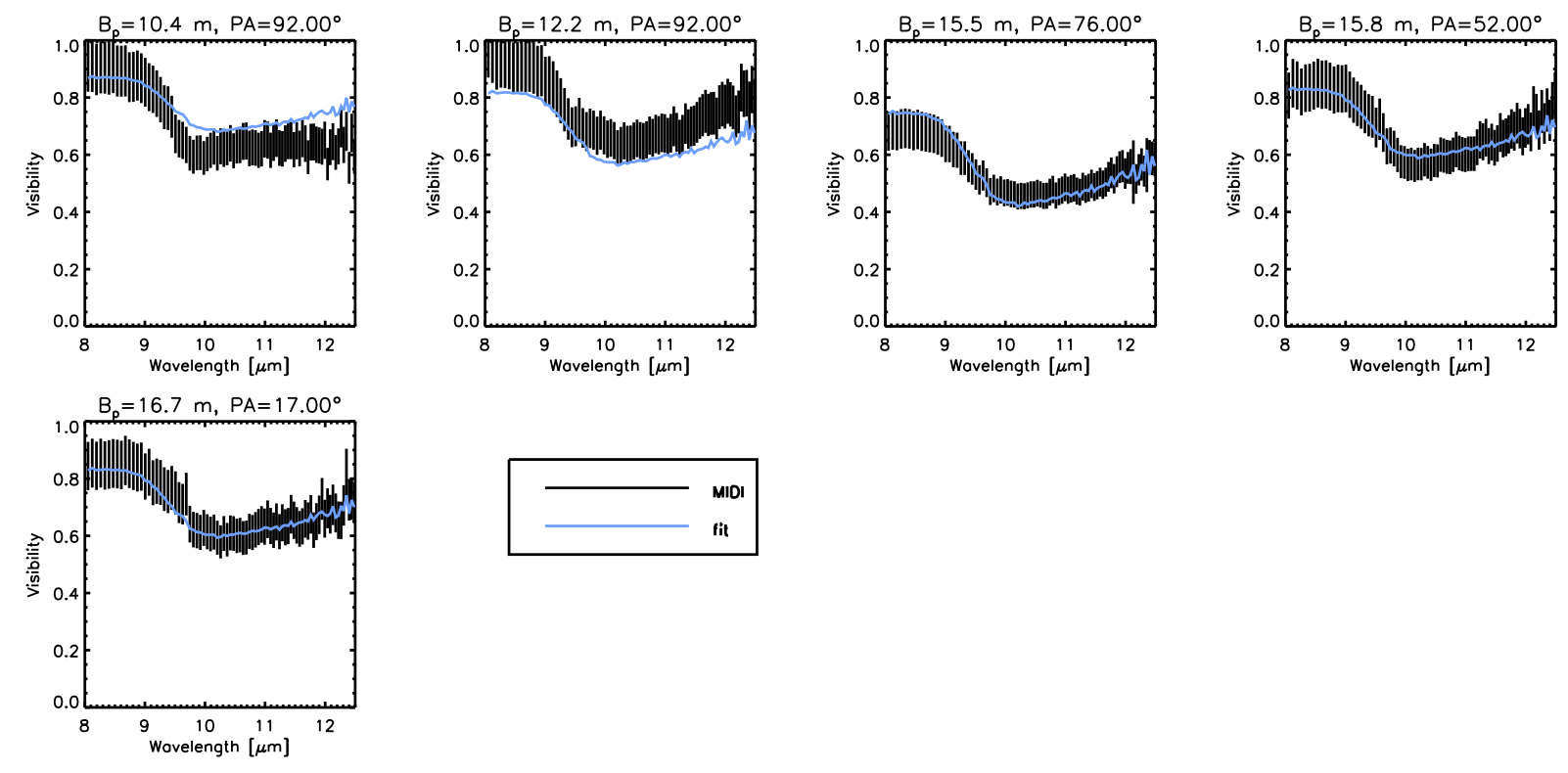

Figure 5. Visibilities dispersed in wavelength of the MIDI observations of $\theta$ Aps. The full line is the best fitting elliptical UD model.

between a Gaussian and a UD profile. Nevertheless the resulting $\chi^{2}$ points toward an elongated envelope in the direction of 120 degree. It might be just a coincidence, but the direction of the elongation is very similar to the one observed in the PACS image. The upcoming VISIR data will be crucial for confirming the possible connection between the Herschel and the MIDI structure. The visibilities modelled with elliptic UD profile are shown in Fig. 5. They all exhibit a decrease around $10 \mu \mathrm{m}$ where the silicate dust feature is located.

\section{CONCLUSIONS AND OUTLOOK}

In this contribution we present a report of the ESO Large program that combines the capability of three extraordinary space and ground facilities: Herschel/PACS, VISIR, and MIDI. The three instruments are used to scan the envelope of a sample of AGB stars in order to trace the origin of asymmetries in the mass-loss process. Preliminary results for the detached shell object U Ant do not show any evidence of big scale asymmetries, confirming the overall sphericity of the envelope of this star. The case of $\theta$ Aps is more complicate. Our preliminary MIDI results show evidence of an elongation. The direction of the elongation is very similar to the one detected in the Herschel/Pacs image (Ref. 27). This might be interpreted as an effect intrinsic to the mass-loss process occurring in the atmosphere of the object, an intermediate scale effect caused by a binary interaction, an effect of the ISM interaction, or just a coincidence. The VISIR observations will clarify the connection between the two detections.

The followup of this work foresees the comparison of the observations with model atmospheres Ref. 25 to characterise the dust and molecular stratification of the envelope of these stars. Moreover we are extending the study to shorter wavelengths. Some of the objects where already observed with AMBER (Paladini et al., in prep.; Wittkowski et al., in prep.), and will be observed by NACO in the next period. These new complementary observations will open a window in the region of the atmosphere where the pulsation is in action.

\section{ACKNOWLEDGMENTS}

This work is supported by Austrian Science Fund FWF under project number AP23006. 


\section{REFERENCES}

[1] H. J. Habing and H. Olofsson, eds., Asymptotic giant branch stars, 2003.

[2] J. M. Chapman, P. Sivagnanam, R. J. Cohen, and A. M. Le Squeren, "OH Maser Emission in the Circumstellar Envelopes of U-Herculis R-Cassiopeiae and W-Hydrae," MNRAS 268, p. 475, May 1994.

[3] M. Szymczak, R. J. Cohen, and A. M. S. Richards, "Full polarization structure of the $\mathrm{OH}$ main-line maser envelopes of W Hydrae," MNRAS 297, pp. 1151-1162, July 1998.

[4] K.-H. Hofmann, Y. Balega, T. Blöcker, and G. Weigelt, "A multi-wavelength study of the oxygen-rich AGB star CIT 3: Bispectrum speckle interferometry and dust-shell modelling," A\&A 379, pp. 529-539, Nov. 2001.

[5] B. Lopez, W. C. Danchi, M. Bester, D. D. S. Hale, E. A. Lipman, J. D. Monnier, P. G. Tuthill, C. H. Townes, C. G. Degiacomi, T. R. Geballe, L. J. Greenhill, P. Cruzalebes, J. Lefevre, D. Mekarina, J. A. Mattei, D. Nishimoto, and P. W. Kervin, "Nonspherical Structures and Temporal Variations in the Dust Shell of O Ceti Observed with a Long Baseline Interferometer at 11 Microns," ApJ 488, p. 807, Oct. 1997.

[6] J. M. Winters, T. Le Bertre, K. S. Jeong, L.-Å. Nyman, and N. Epchtein, "Mass-loss from dusty, low outflow-velocity AGB stars. I. Wind structure and mass-loss rates," A $\mathscr{E} A$ 409, pp. 715-735, Oct. 2003.

[7] C. Kahane and M. Jura, "Bipolar flow in a slowly expanding circumstellar envelope around X Herculis.," A\&A 310, pp. 952-960, June 1996.

[8] G. R. Knapp, K. Young, E. Lee, and A. Jorissen, "Multiple Molecular Winds in Evolved Stars. I. A Survey of CO (2-1) and CO (3-2) Emission from 45 Nearby AGB Stars," ApJ 117, p. 209, July 1998.

[9] P. Bergman, F. Kerschbaum, and H. Olofsson, "The circumstellar CO emission of RV Bootis. Evidence for a Keplerian disk?," AE\&A 353, pp. 257-263, Jan. 2000.

[10] A. Jorissen, A. Frankowski, B. Famaey, and S. van Eck, "Spectroscopic binaries among Hipparcos M giants. III. The eccentricity - period diagram and mass-transfer signatures," A $\because A$ 498, pp. 489-500, May 2009.

[11] H. Olofsson, P. Bergman, R. Lucas, K. Eriksson, B. Gustafsson, and J. H. Bieging, "A high-resolution study of episodic mass loss from the carbon star TT Cygni," A $\mathscr{E} A$ 353, pp. 583-597, Jan. 2000.

[12] P. G. Tuthill, J. D. Monnier, W. C. Danchi, and B. Lopez, "Smoke Signals from IRC +10216. I. Milliarcsecond Proper Motions of the Dust," ApJ 543, pp. 284-290, Nov. 2000.

[13] G. Weigelt, Y. Y. Balega, K.-H. Hofmann, and T. Preibisch, "Diffraction-limited bispectrum speckle interferometry of the Herbig Be star R Mon," A $\& A$ 392, pp. 937-943, Sept. 2002.

[14] M. A. T. Groenewegen, C. Waelkens, M. J. Barlow, F. Kerschbaum, P. Garcia-Lario, J. Cernicharo, J. A. D. L. Blommaert, J. Bouwman, M. Cohen, N. Cox, L. Decin, K. Exter, W. K. Gear, H. L. Gomez, P. C. Hargrave, T. Henning, D. Hutsemékers, R. J. Ivison, A. Jorissen, O. Krause, D. Ladjal, S. J. Leeks, T. L. Lim, M. Matsuura, Y. Nazé, G. Olofsson, R. Ottensamer, E. Polehampton, T. Posch, G. Rauw, P. Royer, B. Sibthorpe, B. M. Swinyard, T. Ueta, C. Vamvatira-Nakou, B. Vandenbussche, G. C. van de Steene, S. van Eck, P. A. M. van Hoof, H. van Winckel, E. Verdugo, and R. Wesson, "MESS (Mass-loss of Evolved StarS), a Herschel key program," A $\mathscr{\ddots} A$ 526, p. A162, Feb. 2011.

[15] F. Kerschbaum, T. Lebzelter, and L. Mekul, "Bolometric corrections for cool giants based on near-infrared photometry," A\&BA 524, p. A87, Dec. 2010.

[16] D. Ladjal, M. J. Barlow, M. A. T. Groenewegen, T. Ueta, J. A. D. L. Blommaert, M. Cohen, L. Decin, W. De Meester, K. Exter, W. K. Gear, H. L. Gomez, P. C. Hargrave, R. Huygen, R. J. Ivison, C. Jean, F. Kerschbaum, S. J. Leeks, T. L. Lim, G. Olofsson, E. Polehampton, T. Posch, S. Regibo, P. Royer, B. Sibthorpe, B. M. Swinyard, B. Vandenbussche, C. Waelkens, and R. Wesson, "Herschel PACS and SPIRE imaging of CW Leonis," A\&A 518, p. L141, July 2010.

[17] A. Jorissen, A. Mayer, S. van Eck, R. Ottensamer, F. Kerschbaum, T. Ueta, P. Bergman, J. A. D. L. Blommaert, L. Decin, M. A. T. Groenewegen, J. Hron, W. Nowotny, H. Olofsson, T. Posch, L. O. Sjouwerman, B. Vandenbussche, and C. Waelkens, "X Herculis and TX Piscium: two cases of ISM interaction with stellar winds observed by Herschel," A\&A 532, p. A135, Aug. 2011.

[18] W. E. C. J. van der Veen and H. J. Habing, "The IRAS two-colour diagram as a tool for studying late stages of stellar evolution," A\&\&A 194, pp. 125-134, Apr. 1988.

[19] D. Klotz, S. Sacuto, F. Kerschbaum, C. Paladini, H. Olofsson, and J. Hron, "The geometry of the close environment of SV Piscium as probed by VLTI/MIDI," A $E A$ A 541, p. A164, May 2012. 
[20] J. Bergeat, A. Knapik, and B. Rutily, "The effective temperatures of carbon-rich stars," $A \& A$ 369, pp. 178209, Apr. 2001.

[21] J. Bergeat and L. Chevallier, "The mass loss of C-rich giants," A $\& A$ 429, pp. 235-246, Jan. 2005.

[22] F. L. Schöier, M. Lindqvist, and H. Olofsson, "Properties of detached shells around carbon stars. Evidence of interacting winds," $A \mathscr{E} A$ 436, pp. 633-646, June 2005.

[23] N. N. Samus, O. V. Durlevich, and et al., "General Catalogue of Variable Stars (Samus+ 2007-2012)," VizieR Online Data Catalog 1, p. 2025, Jan. 2009.

[24] R. Lachaume, "On marginally resolved objects in optical interferometry," A\&A 400, pp. 795-803, Mar. 2003.

[25] S. Höfner, R. Gautschy-Loidl, B. Aringer, and U. G. Jørgensen, "Dynamic model atmospheres of AGB stars. III. Effects of frequency-dependent radiative transfer," A\&SA 399, pp. 589-601, Feb. 2003.

[26] L. Mattsson, R. Wahlin, and S. Höfner, "Dust driven mass loss from carbon stars as a function of stellar parameters. I. A grid of solar-metallicity wind models," A $\& A$ 509, p. A14, Jan. 2010.

[27] N. L. J. Cox, F. Kerschbaum, A.-J. van Marle, L. Decin, D. Ladjal, A. Mayer, M. A. T. Groenewegen, S. van Eck, P. Royer, R. Ottensamer, T. Ueta, A. Jorissen, M. Mecina, Z. Meliani, A. Luntzer, J. A. D. L. Blommaert, T. Posch, B. Vandenbussche, and C. Waelkens, "A far-infrared survey of bow shocks and detached shells around AGB stars and red supergiants," A $E A$ 537, p. A35, Jan. 2012.

[28] H. Olofsson, D. González Delgado, F. Kerschbaum, and F. L. Schöier, "Mass loss rates of a sample of irregular and semiregular M-type AGB-variables," A\& A 391, pp. 1053-1067, Sept. 2002. 